\title{
Extratos de Moringa oleifera e Vernonia sp. sobre Candida albicans e Microsporum canis isolados de cães e gatos e análise da toxicidade em Artemia sp.
}

\author{
Moringa oleifera and Vernonia sp. extracts against Candida albicans and Microsporum \\ canis isolates from dogs and cats and analysis of toxicity to Artemia sp.
}

\author{
Marcos Fábio Gadelha Rocha ${ }^{\mathrm{I}}$ II Franscisco Léo Nascimento de Aguiar \\ Raimunda Sâmia Nogueira Brilhante ${ }^{\mathrm{I}^{*}}$ Rossana de Aguiar Cordeiro ${ }^{\mathrm{II}}$ \\ Carlos Eduardo Cordeiro Teixeira ${ }^{\mathrm{II}}$ Débora de Souza Collares Maia Castelo-Branco ${ }^{\mathrm{II}}$ \\ Manoel de Araújo Neto Paiva ${ }^{I}$ João Paulo Otaviano Zeferino ${ }^{\mathrm{I}}$ Jair Mafezoli $^{\mathrm{II}}$ \\ Célia Maria de Souza Sampaio ${ }^{\mathrm{I}}$ Francisco Geraldo Barbosa ${ }^{\mathrm{III}}$ José Júlio Costa Sidrim ${ }^{\mathrm{II}}$
}

\section{RESUMO}

O aumento da incidência das infecções fúngicas, bem como o registro crescente de resistência e falha terapêutica, têm impulsionado a realização de estudos de prospecção de fitoquímicos com propriedades antifúngicas. Diante do exposto, o presente estudo investigou o potencial antifúngico de extratos de Baccharis ligustrina, B. schultzii, Croton jacobinensis, Licania rigida, Moringa oleifera, Vernonia sp. $e$ V. brasiliana, e de óleos essenciais de Lippia alba (Quimiotipos $1,2,3$ e 4) e Ocimum gratissimum. Inicialmente, foi realizada uma avaliação qualitativa da atividade antifúngica de cada amostra por meio do método de difusão em ágar, frente a cepas de Candida albicans e Microsporum canis, mostrando que apenas os extratos de M. oleifera $(M L F-C)$ e Vernonia $s p$. (TVS-H) apresentaram atividade frente a C. albicans e M. canis, com halos de inibição $=10 \mathrm{~mm}$. Também foram determinadas a concentração inibitória mínima (CIM), frente a 12 cepas de $\boldsymbol{C}$. albicans e M. canis, e a toxicidade aguda de MLF-C e TVS-H, através de protocolos do Clinical and Laboratory Standards Institute (CLSI) e ensaio com Artemia sp., respectivamente. A CIM (80\%) de MLF-C e TVS-H variou de 0,156 a 2,5 mg $\mathrm{mL}^{-1}$ frente C. albicans e de 0,039 a 1,25 e 0,039 a $0,625 \mathrm{mg}^{\mathrm{mL}} \mathrm{L}^{-1}$ para M. canis, respectivamente. A CIM (100\%) de MLF-C e TVS-H variou de 0,625 a >2,5mg $m L^{-1}$ frente C. albicans é de 0,039 a 2,5 e 0,078 a 1,25mg $\mathrm{mL}^{-1}$ para M. canis, respectivamente. As doses letais $\left(D L_{50}\right)$ para o $M L F$ C e TVS-H foram de 201,09 e 204,17 mg $\mathrm{mL}^{-1}$, respectivamente, sendo, portanto, demonstrada a baixa toxicidade desses extratos. Os extratos de M. oileifera e Vernonia sp. apresentaram atividade antifúngica frente cepas de $\boldsymbol{C}$. albicans $e$ M. canis, abrindo a perspectiva de estudos para caracterização dos seus componentes bioativos.
Palavras-chave: Atividade antifúngica, extratos vegetais, Moringa oleifera, Vernonia sp., Candida albicans, Microsporum canis.

\section{ABSTRACT}

The increase in the incidence of fungal infections and the frequent report of resistance and therapeutic failure has promoted the performance of phytochemical screening for compounds with antifungal properties. Based on this, the present study investigated the antifungal potential of extracts of Baccharis ligustrina, B. schultzii, Croton jacobinensis, Licania rigida, Moringa oleifera, Vernonia sp. and V. brasiliana and of essential oils of Lippia alba (Chemotypes 1, 2, 3 and 4) and Ocimum gratissimum. Initially, a qualitative evaluation of the antifungal activity of each vegetal sample against strains of Candida albicans and Microsporum canis, through the agar diffusion method, was performed. Extracts of M. oleifera (MLFC) and Vernonia sp. (TVS-H) presented activity against $\boldsymbol{C}$. albicans and $M$. canis with inhibition halos $=10 \mathrm{~mm}$. Then, minimum inhibitory concentrations (MICs) for MLF-C and TVS-H against 12 strains of $\boldsymbol{C}$. albicans and M. canis were determined through the methodology established by the Clinical and Laboratory Standards Institute (CLSI), and acute toxicity tests against Artemia sp. were performed for both extracts. MICs (80\%) for MLF-C and TVS-H varied from 0.156 to $2.5 \mathrm{mg}$ $\mathrm{mL}^{-1}$ against $\boldsymbol{C}$. albicans and from 0.039 to $1.25 \mathrm{mg} \mathrm{mL}^{-1}$ and 0.039 to $0.625 \mathrm{mg} \mathrm{mL}^{-1}$ against $\mathbf{M}$. canis, respectively. MICs (100\%) for MLF-C and TVS-H varied from 0.625 to $>2.5 \mathrm{mg}$ $\mathrm{mL}^{-1}$ for C. albicans and from 0.039 to $2.5 \mathrm{mg}^{\mathrm{mL}} \mathrm{L}^{-1}$ and 0.078 to $1.25 \mathrm{mg} \mathrm{mL}^{-1}$ against $M$. canis, respectively. Lethal doses $\left(D L_{50}\right)$ of MLF-C and TVS-H were 201.09 and $204.17 \mathrm{mg} \mathrm{mL}^{-1}$,

'Programa de Pós-graduação em Ciências Veterinárias, Faculdade de Veterinária, Universidade Estadual do Ceará (UFCE), Fortaleza, CE, Brasil. "Programa de Pós-graduação em Microbiologia Médica, Departamento de Patologia e Medicina Legal, Faculdade de Medicina, Centro Especializado em Micologia Médica, UFCE. *Endereço para correspondência: Rua Barão de Canindé, 210, Montese, 60425-540, Fortaleza, CE, Brasil. E-mail: brilhante@ufc.br.

"I'Departamento de Química Orgânica e Inorgânica, Centro de Ciências, UFCE, Fortaleza, CE, Brasil. 
respectively, being, therefore, demonstrated the low toxicity of these extracts. M. oleifera and Vernonia sp. extracts presented in vitro antifungal activity against $\boldsymbol{C}$. albicans and M. canis, creating perspectives for the development of studies on the characterizations of their bioactive components.

Key words: Antifungal activity, vegetal extracts, Moringa oleifera, Vernonia sp., Candida albicans, Microsporum canis.

\section{INTRODUÇÃO}

Com o aumento da frequência de infecções fúngicas em seres humanos, a realização de pesquisas visando suscitar novas alternativas terapêuticas frente a fungos de importância médico-veterinária ganhou força. As plantas são tradicionalmente usadas por populações de todos os continentes no tratamento de doenças desde a antiguidade, sendo fonte importante de produtos naturais biologicamente ativos, muitos dos quais constituem modelos para a síntese de um grande número de fármacos, revelando nestes produtos alta diversidade em termos de estrutura e de propriedades físico-químico-biológicas (SIMÕES et al., 2004; FONTENELLE et al., 2007).

Segundo BRILHANTE et al. (2003), nos animais domésticos, as dermatofitoses, consideradas como micoses superficiais, possuem grande interesse pelo seu potencial zoonótico. Microsporum canis é o responsável pela maioria de casos de micoses em animais de estimação e o mais frequente dermatófito zoofílico de humanos, em diversas áreas urbanas (SIDRIM et al., 2004; FRIAS; KOZUSNYANDREANI, 2010). Quanto às micoses causadas por leveduras em animais domésticos, destacam-se os fungos do gênero Candida, que podem tornar-se patógenos em decorrência de desequilíbrio nas proteções física, química e imunológica de seres humanos e animais, levando assim a enfermidades, as quais requerem investimentos no tratamento, principalmente na sua forma sistêmica (MORAN et al., 2010).

Segundo SOEJARTO (1996), apesar do aumento dos estudos sobre plantas medicinais, somente 15 a $17 \%$ destas foram estudadas quanto ao seu potencial medicinal. Considerando que o Brasil pertence a uma minoria de países ditos megadiversos, com cerca de $20 \%$ da flora mundial, esse número poderia ser bem maior (FUNARI \& FERRO, 2005). Emerge deste cenário o estudo de plantas como fonte de substâncias bioativas, as quais podem subsidiar novos compostos na terapêutica antifúngica, por meio da bioprospecção (RUKAYADI et al., 2008).
O objetivo inicial deste trabalho foi identificar o potencial antifúngico de extratos de Baccharis ligustrina, B. schultzii, Croton jacobinensis, Licania rigida, Moringa oleifera, Vernonia sp. e V. brasiliana, e de óleos essenciais de Lippia alba (Quimiotipos 1, 2, 3 e 4) e Ocimum gratissimum em cepas de $\boldsymbol{C}$. albicans e $\boldsymbol{M}$. canis, através do método de difusão em ágar. A seguir, buscou-se determinar a concentração inibitória mínima (CIM) e toxicidade dos produtos com atividade antifúngica no screening inicial.

\section{MATERIAL E MÉTODOS}

Amostras testadas

Os extratos de B. ligustrina (vassourinha), B. schultzii (vassoura), C. Jacobinensis (marmeleiro), L. rigida (oiticica), M. oleifera (baqueta), Vernonia sp. e $\boldsymbol{V}$. brasiliana (assa-peixe), assim como os óleos essenciais de $\boldsymbol{L}$. alba (cidreira) (Quimiotipos 1, 2, 3 e 4) e $\boldsymbol{O}$. gratissimum (alfavaca) foram preparados com base em trabalhos anteriores (FONTENELLE et al., 2007; LEITE et al., 2009). Considerando que o screening inicial mostrou que apenas os extratos de flores de M. oleifera (MLF-C) e de talos de Vernonia sp. (TVS-H) tiveram atividade frente a $\boldsymbol{C}$. albicans e M. canis, ao longo deste trabalho, serão abordados somente estes dois extratos.

Preparação do extrato MFL-C de flores de M. oleifera Lam.

As flores de M. oleifera foram coletadas a partir de espécimes cultivados em Fortaleza, Ceará. Depois de secas em estufa a $40^{\circ} \mathrm{C}$, as flores $(50 \mathrm{~g})$ foram submetidas a três extrações sucessivas por maceração a frio com clorofórmio P.A (1L) em intervalos de $24 \mathrm{~h}$. Após filtração e evaporação do solvente sob pressão reduzida em evaporador rotativo, obteve-se $14,73 \mathrm{~g}$ do extrato, o qual foi denominado MFL-C.

Preparação do extrato TVS-H de talos de Vernonia sp.

Os talos de Vernonia sp. foram coletados na serra de São Miguel no município de Itapipoca, Ceará. Depois de secos em estufa a $40^{\circ} \mathrm{C}$, os talos $(60,5 \mathrm{~g})$ foram submetidos a três extrações sucessivas por maceração a frio com hexano P.A $(250 \mathrm{~mL})$ em intervalos de $24 \mathrm{~h}$. Após filtração e evaporação do solvente sob pressão reduzida em evaporador rotativo, obteve-se $0,341 \mathrm{~g}$ do extrato, o qual foi denominado TVS-H.

Cepas fúngicas

Neste estudo, foram utilizadas cepas de $\boldsymbol{C}$. albicans $(\mathrm{n}=12)$, oriundas de microbiota oral de cães, e 
de M. canis $(\mathrm{n}=12)$, oriundas de quadros de dermatofitose felina. Estes fungos pertencem à Micoteca do Centro Especializado em Micologia Médica (CEMM) da Universidade Federal do Ceará. Para controle de qualidade, foram utilizadas as cepas C. albicans (ATCC 10231), C. parapsilosis (ATCC 22019), C. krusei (ATCC 6528) e M. canis (CEMM 0103-088).

Método de difusão em ágar

Os extratos de B. ligustrina, B. schultzii, $\boldsymbol{C}$. jacobinensis, L. rigida, M. oleifera, Vernonia sp. e $V$. brasiliana, assim como os óleos essenciais de $\boldsymbol{L}$. alba (Quimiotipos 1, 2, 3 e 4) e O. gratissimum foram inicialmente testados contra as cepas de $\boldsymbol{C}$. albicans $(n=8)$ e M. canis $(n=7)$, por meio do método de difusão em ágar (FONTENELLE et al., 2007). Para controle, foram utilizados: anfotericina $\mathrm{B}\left(1 \mathrm{mg} \mathrm{L}^{-1}\right.$; Sigma Chemical Co.) e griseofulvina $\left(64 \mu \mathrm{g} \mathrm{mL}^{-1}\right.$; Sigma Chemical Co) para as cepas de C. albicans e M. canis, respectivamente. Foi considerada satisfatória a presença de halo de inibição maior ou igual a $10 \mathrm{~mm}$, bem como atividade antifúngica contra ambas as espécies fúngicas testadas (C. albicans e M. canis).

Método de microdiluição em caldo

Para a determinação da concentração inibitória mínima (CIM), foi empregado o método de microdiluição em caldo segundo o documento M27A2 (CLSI, 2002) para cepas de $\boldsymbol{C}$. albicans. Para as cepas de $\boldsymbol{M}$. canis, foi utilizado o protocolo descrito por BRILHANTE et al. (2004). Os extratos vegetais foram avaliados nos intervalos de concentrações de 0,005 a $2,5 \mathrm{mg} \mathrm{mL}^{-1}$. As drogas-controle foram testadas nas concentrações de 0,125 a $64 \mu \mathrm{g} \mathrm{mL} \mathrm{mL}^{-1}$. O ensaio de microdiluição foi realizado em placas contendo 96 poços, em volume total de $200 \mu \mathrm{L}$. As placas foram incubadas a $35^{\circ} \mathrm{C}$ e as leituras foram realizadas após dois dias para $\boldsymbol{C}$. albicans e cinco dias para M. canis. Todos os testes foram realizados em duplicata. A leitura foi feita através de comparação visual com o controle de crescimento fúngico sem droga. Foram considerados os parâmetros de inibição total (CIM 100\%) e parcial (80\%) do crescimento (BRILHANTE et al., 2004; FONTENELLE et al., 2007).

Determinação da toxicidade e da dose letal $\left(\mathrm{DL}_{50}\right)$

A avaliação da toxicidade dos extratos vegetais com atividade antifúngica frente a Artemia sp. foi realizada segundo o método descrito por MEYER (1982). Os extratos com atividade antifúngica foram testados nas seguintes concentrações: 300, 250, 225, $200,175,150,125$ e $100 \mathrm{mg} \mathrm{mL}^{-1}$. Os ensaios foram conduzidos em tubos de ensaio contendo $5 \mathrm{~mL}$ da solução-teste e 10 larvas de Artemia sp. Um grupo controle também foi preparado nas mesmas condições dos tratamentos, mas sem a presença da substânciateste. Para o cálculo da DL 50, foi utilizado o aplicativo Trimmed Spearman-Karber Method Version 1.5. Todos os tratamentos foram realizados em triplicata.

\section{RESULTADOS}

Dentre os extratos testados, apenas aqueles obtidos de M. oleifera e Vernonia sp. mostraram atividade antifúngica por meio do método de difusão em ágar frente a $\boldsymbol{C}$. albicans (3/8) e M. canis (7/7). O extrato TVS-H apresentou atividade frente $\boldsymbol{C}$. albicans, com halos de inibição de $10 \mathrm{~mm}$, bem como frente a $\boldsymbol{M}$. canis, cujos halos de inibição variaram de 12,5 a $28 \mathrm{~mm}$. O extrato MLF-C apresentou atividade frente $\boldsymbol{C}$. albicans e M. canis com halos variando de 13 a $14 \mathrm{~mm}$ e 17,5 a 37mm, respectivamente (Tabela 1).

A CIM 80\% de TVS-H e MLF-C frente $\boldsymbol{C}$. albicans variou de 0,156 a $2,5 \mathrm{mg} \mathrm{mL}^{-1}$, enquanto que ante a cepas de $\boldsymbol{M}$. canis foi de 0,039 a 0,625 e 0,039 a $1,25 \mathrm{mg} \mathrm{mL}^{-1}$, respectivamente. ACIM $100 \%$ de TVS-H e MLF-C frente a $\boldsymbol{C}$. albicans variou de 0,625 a $>2,5 \mathrm{mg}$ $\mathrm{mL}^{-1}$; enquanto que ante as cepas de $\boldsymbol{M}$. canis foi de 0,078 a 1,25 e 0,039 a $2,5 \mathrm{mg} \mathrm{mL}^{-1}$, respectivamente (Tabela 2). Para o extrato TVS-H, a CL50 estimada foi de $204,17 \mathrm{mg} \mathrm{mL}^{-1}$, com intervalo de confiança de $95 \%$ variando entre 211,28 a $197,31 \mathrm{mg} \mathrm{mL}^{-1}$, enquanto que o extrato MLF-C apresentou CL50 estimada de 201,09mg $\mathrm{mL}^{-1}$, com intervalo de confiança de $95 \%$ variando de 216,25 a $187 \mathrm{mg} \mathrm{mL}^{-1}$.

\section{DISCUSSÃO}

M. oleifera é uma das espécies mais comuns da família Moringaceae. Estudos recentes mostraram a atividade antifúngica de extratos e óleo essencial de M. oleifera, frente a diversas espécies fúngicas, levantando o interesse da comunidade científica (CHUANG et al., 2007). Extrato de sementes e o óleo essencial de M. oleifera possuem atividade inibitória frente aos dermatófitos Epidermophyton floccosum, Trichophyton rubrum, T. mentagrophytes $\mathrm{e}$ M. canis, mostrando CIMs variando de $0,1 \mathrm{mg} \mathrm{mL}^{-1}$ a $1,6 \mathrm{mg} \mathrm{mL}^{-1}$ para o óleo essencial e de $0,156 \mathrm{mg} \mathrm{mL}^{-1}$ a $2,5 \mathrm{mg} \mathrm{mL}^{-1}$ para o extrato de semente. $O$ extrato de folha, por sua vez, possui pouca atividade inibitória, com CIMs superiores a $10 \mathrm{mg} \mathrm{mL}^{-1}$ (CHUANG et al., 2007). No presente estudo, foi mostrado de forma inédita que o extrato de flores de $\boldsymbol{M}$. oleifera também é capaz de inibir tanto o crescimento de $\boldsymbol{M}$. canis como o de $\boldsymbol{C}$. 
Tabela 1 - Atividade antifúngica de extratos de Vernonia sp. (TVS-H) e de Moringa oleifera (MLF-C) na concentração de $10 \mathrm{mg} \mathrm{mL}^{-1}$ frente a $\boldsymbol{C}$. albicans e M. canis através do método de difusão em ágar.

\begin{tabular}{cccccccc}
\hline \multicolumn{7}{c}{ Zona de inibição (mm) } \\
\hline C. albicans CEMM & TVS-H & MFL-C & $\begin{array}{c}\text { ANFB } \\
\left(1 \mathrm{mg} \mathrm{mL}^{-1}\right)\end{array}$ & M. canis CEMM & TVS-H & MFL-C & GRS $\left(64 \mu \mathrm{g} \mathrm{mL}^{-1}\right)$ \\
$01-2-165$ & - & - & 19,0 & $01-3-179$ & 18,0 & 30,0 & 40,0 \\
$02-1-070$ & 10,0 & 14,0 & 19,0 & $01-3-088$ & 18,5 & 17,5 & 48,0 \\
$01-5-004$ & 10,0 & 14,0 & 20,0 & $01-3-162$ & 12,5 & 22,5 & 45,0 \\
$01-5-005$ & 10,0 & 13,0 & 19,0 & $01-3-165$ & 28,0 & 37,0 & 41,0 \\
$03-2-033$ & - & - & 17,0 & $01-5-190$ & 12,5 & 18,0 & 40,0 \\
$01-2-078$ & - & - & 16,0 & $01-3-164$ & 17,0 & 21,0 & 39,0 \\
$01-4-037$ & - & - & 16,0 & $01-3-168$ & 16,0 & 19,0 & 41,0 \\
$01-5-006$ & - & - & 17,0 & & & & \\
\hline
\end{tabular}

- sem inibição do crescimento. ANFB (Anfotericina B). GRS (Grizeofulvina).

albicans. A partir desses, achados ficou comprovado que diferentes partes desta planta apresentam atividade antifúngica contra importantes fungos patogênicos, tanto para o homem como para animais. Ademais, estes dados in vitro dão subsídio científico para o delineamento de estudos in vivo, com vistas à obtenção de um possível fitoterápico com atividade antifúngica.

Vernonia sp. compreende espécies de plantas herbáceas comuns em resquícios de Mata Atlântica, com conhecida atividade antifúngica frente ao fitopatógeno Penicillium citrinum (FREIRE et al., 1996). Estudo realizado por Ogundare et al. (2006) mostrou que extratos obtidos da casca de $\boldsymbol{V}$. tenoreana inibiram o crescimento de $\boldsymbol{C}$. albicans, $\boldsymbol{A}$. flavus e $\boldsymbol{A}$. niger, com CIMs de $15 \mathrm{mg} \mathrm{mL}^{-1}$.
Este estudo utilizou cepas de $\boldsymbol{C}$. albicans isoladas da microbiota oral de cães e de $\boldsymbol{M}$. canis isolados de quadros de dermatofitose felina. A partir de comparações interespécie fúngica, não se observaram diferenças estatísticas no perfil de sensibilidade das cepas frente aos extratos MLF-C e TVS-H. Este trabalho adiciona dados importantes sobre o potencial antifúngico dos extratos de M. oleifera e Vernonia sp. frente aos patógenos $\boldsymbol{C}$. albicans e $\boldsymbol{M}$. canis, sendo este o primeiro relato de atividade antifúngica de extrato de Vernonia sp. frente a este dermatófito. Os dados obtidos abrem perspectiva para a realização de estudos fitoquímicos guiados, a fim de se identificar os compostos responsáveis pela atividade antifúngica detectada. Estudos prévios têm apontado

Tabela 2 - Concentração inibitória mínima dos extratos brutos de Vernonia sp. (TVS-H) e de Moringa oleifera (MLF-C) frente a C. albicans e M. canis por meio do método de microdiluição em caldo.

\begin{tabular}{|c|c|c|c|c|c|c|c|c|c|}
\hline \multirow{3}{*}{ C. albicans CEMM } & \multicolumn{2}{|c|}{ TVS-H } & \multicolumn{2}{|c|}{ MLF-C } & \multirow{3}{*}{ M. canis CEMM } & \multicolumn{2}{|c|}{ TVS-H } & \multicolumn{2}{|c|}{ MLF-C } \\
\hline & MIC (1 & $\left.m L^{-1}\right)$ & MIC ( & $\left.\mathrm{mL}^{-1}\right)$ & & MIC & $\left.\mathrm{mL}^{-1}\right)$ & $\mathrm{MIC}(\mathrm{r}$ & $\left.\mathrm{mL}^{-1}\right)$ \\
\hline & $100 \%$ & $80 \%$ & $100 \%$ & $80 \%$ & & $100 \%$ & $80 \%$ & $100 \%$ & $80 \%$ \\
\hline $02-1-070$ & 1,25 & 0,625 & 1,25 & 0,625 & $03-2-023$ & 0,156 & 0,078 & 0,078 & 0,039 \\
\hline 01-4-037 & 1,25 & 0,625 & 1,25 & 0,625 & 01-4-080 & 0,312 & 0,156 & 1,25 & 0,625 \\
\hline $01-2-184$ & 1,25 & 0,625 & $>2,5$ & 0,625 & $01-3-179$ & 0,156 & 0,078 & 0,039 & 0,039 \\
\hline $01-5-004$ & 0,625 & 0,312 & 0,625 & 0,312 & $01-3-162$ & 0,312 & 0,156 & 2,5 & 1,25 \\
\hline $01-5-005$ & 1,25 & 0,625 & 0,625 & 0,312 & $01-3-168$ & 0,625 & 0,312 & 1,25 & 0,625 \\
\hline $01-5-006$ & 2,5 & 1,25 & $>2,5$ & 0,156 & $01-3-164$ & 0,078 & 0,039 & 0,078 & 0,039 \\
\hline $01-5-012$ & 2,5 & 1,25 & $>2,5$ & 2,5 & $01-3-165$ & 0,156 & 0,078 & 0,156 & 0,078 \\
\hline $02-2-170$ & 1,25 & 0,312 & $>2,5$ & 0,625 & $01-3-088$ & 0,156 & 0,078 & 0,625 & 0,312 \\
\hline $02-2-261$ & 0,625 & 0,156 & $>2,5$ & 0,156 & $01-5-188$ & 1,25 & 0,625 & 1,25 & 0,625 \\
\hline $03-2-033$ & 2,5 & 1,25 & $>2,5$ & 0,312 & $01-3-167$ & 1,25 & 0,625 & 0,078 & 0,039 \\
\hline $01-2-078$ & $>2,5$ & 2,5 & 1,25 & 0,625 & $01-3-170$ & 0,625 & 0,3125 & 0,3125 & 0,156 \\
\hline $01-3-077$ & 2,5 & 1,25 & $>2,5$ & 0,312 & $02-6-074$ & 0,156 & 0,078 & 0,625 & 0,312 \\
\hline
\end{tabular}

Ciência Rural, v.41, n.10, out, 2011. 
flavonoides, alcaloides e taninos, bem como triterpenoides, saponinas, coumarinas e antracnonas, como compostos importantes na composição química desses produtos (BRAGA et al., 2006).

Embora já tenha sido relatada atividade antifúngica em extratos de Baccharis spp. (MORALES et al., 2008), $\boldsymbol{C}$.jacobinensis (RAMOS et al., 2009), $\boldsymbol{C}$. jacobinensis, Lippia alba (AGUIAR et al., 2008) (Quimiotipos 1, 2, 3 e 4) e O. gratissimum (FARIA et al., 2006), o presente estudo mostrou que os extratos oriundos desses vegetais apresentaram reduzida ou nula atividade inibitória frente aos modelos biológicos empregados, haja vista o reduzido tamanho dos halos de inibição encontrados $(<10 \mathrm{~mm})$. No delineamento desta pesquisa, foi estabelecido que somente aqueles produtos que apresentassem efeito inibitório concomitante para o dermatófito e para a levedura seriam considerados com potencialidade antifúngica. Este fato explica porque, apesar de já ter sido descrita atividade antifúngica para muito dos produtos testados, a presente pesquisa não retificou tal efeito. Por outro lado, o estabelecimento deste rígido critério foi importante, porque dentre os compostos avaliados foram identificados os extratos de flores de M. oleifera e talos de Vernonia sp. com potencialidade antifúngica.

A toxicidade reduzida dos extratos de $\boldsymbol{M}$. oleifera e Vernonia sp. no modelo biológico de Artemia sp., que possui boa correlação com o modelo murino (LOGARTO et al., 2001), reforça o potencial fitoterápico antifúngico destes produtos naturais. Por fim, destaca-se que, associado ao efeito contra M. canis e $\boldsymbol{C}$. albicans, estes produtos apresentaram baixa toxicidade, estimulando assim a continuação desta pesquisa, através de experimentos in vivo visando à obtenção de produtos que possam ser utilizados na medicina veterinária.

\section{CONCLUSÃO}

Os extratos de M. oleifera e Vernonia sp. possuem atividade antifúngica ante as cepas de $\boldsymbol{C}$. albicans e $\boldsymbol{M}$. canis in vitro e baixa toxicidade no modelo de Artemia sp., abrindo a perspectiva de estudos para caracterização química dos componentes bioativos, que devem ser testados isoladamente contra estes fungos e avaliados em protocolos de toxicidade subaguda, aguda e crônica, para posteriormente serem usados em modelos experimentais in vivo.

\section{AGRADECIMENTOS}

Este trabalho teve auxílio financeiro do Conselho Nacional de Desenvolvimento Científico e Tecnológico (CNPq; Processos: 302574/2009-3 e 562296/2010-7).

\section{REFERÊNCIAS}

AGUIAR, J.S. et al. Atividade antimicrobiana de Lippia alba (Mill.) N. E. Brown (Verbenaceae). Braz J Pharmacogn, v.18, n.3, p.436-440, 2008. Disponível em: <http:// www.scielo.br/scielo.php? script $=$ sci_arttext\&pid=S0102$695 \times 2008000300018 \& \operatorname{lng}=e n \& n r m=i s o \& t \operatorname{lng}=p t<$. Acesso em: 17 Jun. 2011. doi: 10.1590/S0102-695X2008000300018.

BRAGA, F.G. et al. Antileishmanial and antifungal activity of plants used in traditional medicine in Brazil. $\mathbf{J}$ Ethnopharmacol, v.111, n.4, p.396-402, 2006. Disponível em: <http://www.sciencedirect.com/science/article/pii/ S0378874106006374>. Acesso em: 4 Mai. 2007. doi:10.1016/ j.jep.2006.12.006.

BRILHANTE, R.S.N. et al. Tinea capitis in a dermatology center in the city of Fortaleza, Brazil: the role of Trichophyton tonsurans. Int J Dermatol, v.43, n.4, p.575-579, 2004. Disponível em: 〈www.ncbi.nlm.nih.gov/pubmed/15304180〉. Acesso em: 3 Ago. 2004 doi: 10.1111/j.13654632.2004.02150.x.

BRILHANTE, R.S.N. et al. High rate of Microsporum canis feline and canine dermatophytoses in Northeast Brazil: epidemiological and diagnostic features. Mycopathologia, v.156, n.4, p.303-308, 2003. Disponível em: <http://www.springerlink.com/content/ m054222j50235403/>. Acesso em: 17 Jun. 2011. doi: 10.1023/ B:MYCO.0000003582.67122.69.

CHUANG, P.H. et al. Antifungal activity of crude extracts and essential oil of Moringa oleifera Lam. Bioresour Technol, v.98, n.1, p.232-236, 2007. Disponível em: <http:// www.sciencedirect.com/science/article/pi / S0960852405005286>. Acesso em: 6 Jan. 2007. doi:10.1016/ j.biortech.2005.11.003.

FARIA T.J. et al. Antifungal activity of essential oil isolated from Ocimum gratissimum L. (eugenol chemotype) against phytopathogenic fungi. Braz Arch Biol Techn, v.49, p 867871, 2006. Disponível em: <www.scielo.br/pdf/babt/v49n6/ a02v49n6.pdf $>$. Acesso em: 1 Mar. 2011. doi: 10.1590/S151689132006000700002 .

FONTENELLE, R.O. et al. Chemical composition, toxicological aspects and antifungal activity of essential oil from Lippia sidoides Cham. J Antimicrob Chemother, v.59, n.5, p.934-940, 2007. Disponível em: <http:// jac.oxfordjournals.org/content/59/5/934.abstract $>$. Acesso em: 8 Fev. 2007. doi:10.1093/jac/dkm066.

FREIRE, M.F.I; ABREU, H.S. Extratos de raízes de Vernonia scorpioides com potencial antibiótico contra Penicillium citrinum. In : CONGRESSO LATINO AMERICANO DE CIÊNCIA DO SOLO, 13.; REUNIÃO BRASILEIRA DE BIOLOGIA DO SOLO; SIMPÓSIO BRASILEIRO SOBRE MICROBIOLOGIA DO SOLO,4.; REUNIÃO BRASILEIRA SOBRE MICORRIZAS, 6.; REUNIÃO BRASILEIRA DE MANEJO E CONSERVAÇÃO DO SOLO E DA ÁGUA, 11., 1996, Águas de Lindóia, SP. Anais... Águas de Lindóia: Sociedade Latino Americana de Ciência do Solo. 4 a 8 de agosto de 1996, SP, Brasil. CD.

FRIAS, D.F.R.; KOZUSNY-ANDREANI, D.I. Utilização de extratos de plantas medicinais e óleo de Eucaliptus no controle in vitro de Microsporum canis. Rev Cubana Plant Med, 
v.15, n.3, p.119-125, 2010. Disponível em: <http:// www.bvs.sld.cu/revistas/pla/vol_15_3_10/pla03310.htm>. Acesso em: 30 Mai. 2011. ISSN: 1028-4796.

FUNARI, C.S.; FERRO, V.O. Uso ético da biodiversidade brasileira: necessidade e oportunidade. Braz J Pharmacogn, v.15, n.2, p.178182, 2005. Disponível em: <http://www.scielo.br/ scielo.php?pid=S0102-695X2005000200018\&script=sci_arttext $>$. Acesso em: 17 Jun. 2011. doi: 10.1590/S0102695X2005000200018.

LEITE, J.J. et al. Chemical composition, toxicity and larvicidal and antifungal activities of Persea americana (avocado) seed extracts. Rev Soc Bras Med Trop, v.24, n.2, p.110-113, 2009. Disponível em: <http://www.scielo.br/pdf/rsbmt/v42n2/ v42n2a03.pdf>. Acesso em: 17 Mai. 2009. doi: 10.1590/S003786822009000200003 .

LOGARTO, P.A. et al. Comparative study of the assay of Artemia salina L. and the estimate of the medium lethal dose (LD50 value) in mice to determine oral acute toxicity of plant extracts. Phytomedicine, v.8, n.5, p.395-400, 2001. Disponível em: <http://www.sciencedirect.com/science/article/ pii/S0944711304700574>. Acesso em: 17 Jun. 2011. doi:10.1078/0944-7113-00044.

MEYER, B.N. et al. Brine shrimp: a convenient general bioassay for active plant constituents. Planta Med, v.45, n.5, p.31-34, 1982. Disponível em: <http://www.ncbi.nlm.nih.gov/pubmed/ 7100305>. Acesso em: 17 Jun. 2011. doi: 10.1055/s-2007971236

MORALES, G. et al. Antimicrobial activity of three Baccharis species used in the traditional medicine of northern Chile. Molecules, v.13, n.4, p.790-794, 2008. Diponível em: <http:/ /www.mdpi.com/1420-3049/13/4/790/>. Acesso em: 1 Out. 2009. doi:10.3390/molecules13040790.

MORAN, C. et al. Comparison of costs, length of stay, and mortality associated with Candida glabrata and Candida albicans bloodstream infections. Am J Infect Control, v.38, n.1, p.78-80, 2010. Disponível em: <http:// www.sciencedirect.com/science/article/pii/ S019665530900755X>. Acesso em: 17 Fev. 2011. doi:10.1016/ j.ajic.2009.06.014.

OGUNDARE, A.O. et al. Antimicrobial activities of Vernonia tenoreana. African J Biotechnology, v.5, n.18, p.1663-1668, 2006. Disponível em: <http://scialert.net/abstract/ ?doi=tasr.2007.145.150>. Acesso em: 17 Jun. 2011. doi: 10.3923/ tasr.2007.145.150.

RAMOS, S.C.S. et al. Antibacterial and cytotoxic properties of some plant crude extracts used in Northeastern folk medicine. Rev Bras Farmacogn, v.19, n.2, p.376-381, 2009. Disponível em: <http://www.scielo.br/scielo.php? pid=S 0102 695X2009000300007\&script=sci_arttext $>$. Acesso em: 20 Mar. 2011. doi: 10.1590/S0102-695X2009000300007.

RUKAYADI, Y. et al. Screening of Thai medicinal plants for anticandidal activity. Mycoses, v.51, n.4, p.308-312, 2008. Disponível em: <http://onlinelibrary.wiley.com/doi/10.1111/ j.1439-0507.2008.01497.x/full>. Acesso em: 4 Mar. 2008. doi: $10.1111 / \mathrm{j} .1439-0507.2008 .01497 . x$.

SIDRIM, J.J.C. et al. Aspectos clínico-laboratoriais das dermatofitoses. In: SIDRIM, J.J.C.; ROCHA, M.F.G. Micologia médica à luz dos autores contemporâneos. Rio de Janeiro: Guanabara Koogan, 2004. Cap. 4, p.135-161.

SIMÕES C.M.O. et al. Farmacognosia: da planta ao medicamento. Porto Alegre/ Florianópolis: UFRGS, UFSC, 2004. p. 821.

SOEJARTO, D.D. Biodiversity prospecting and benefit sharing: perspectives from the field. J Ethnopharmacol, v.51, p.115, 1996. Disponível em: <http://www.sciencedirect.com/ science/article/pii/0378874195013458>. Acesso em: 17 Jun. 2011. doi: 10.1016/0378-8741(95)01345-8. 